\title{
On stress-constrained fail-safe structural optimization considering partial damage
}

\author{
Dou, Suguang; Stolpe, Mathias
}

Published in:

Structural and Multidisciplinary Optimization

Link to article, DOI:

$10.1007 / \mathrm{s} 00158-020-02782-2$

Publication date:

2021

Document Version

Peer reviewed version

Link back to DTU Orbit

Citation (APA):

Dou, S., \& Stolpe, M. (2021). On stress-constrained fail-safe structural optimization considering partial damage. Structural and Multidisciplinary Optimization, 63, 929-933. https://doi.org/10.1007/s00158-020-02782-2

\section{General rights}

Copyright and moral rights for the publications made accessible in the public portal are retained by the authors and/or other copyright owners and it is a condition of accessing publications that users recognise and abide by the legal requirements associated with these rights.

- Users may download and print one copy of any publication from the public portal for the purpose of private study or research.

- You may not further distribute the material or use it for any profit-making activity or commercial gain

- You may freely distribute the URL identifying the publication in the public portal

If you believe that this document breaches copyright please contact us providing details, and we will remove access to the work immediately and investigate your claim 
This is a post-peer-review, pre-copyedit version of an article published in Structural and Multidisciplinary Optimization. The final authenticated version is available online at: DOI: $10.1007 / \mathrm{s} 00158-020-02782-2$

\title{
On stress-constrained fail-safe structural optimization considering partial damage
}

\author{
Suguang Dou • Mathias Stolpe
}

Received: date / Accepted: date

\begin{abstract}
This brief note presents some observations about the modelling of stress constraints in fail-safe structural optimization considering local degradation of member properties. An analytical study of a three-bar example demonstrates the impact of the constraint modelling choices on the optimal design. One notable finding is that degradation of one arbitrary member may cause a worse objective function than complete removal of the member. The observation is similar to the singular optimum issue in topology optimization with stress constraints, but applies also to sizing problems. This deserves special attention and should be considered when modelling fail-safe structural optimization with stress constraints.
\end{abstract}

Keywords Fail-safe structural optimization · Partial damage $\cdot$ Stress constraints $\cdot$ Sizing optimization

\section{Introduction}

In the literature, fail-safe structural optimization has long been developed to achieve fail-safe design by including damage scenarios or failure modes into the design optimization problem, see e.g. Sun et al. (1976); Achtziger and Bendsøe

This work was supported by the Independent Research Fund Denmark under grant number 7017-00084A: Fail-Safe Structural Optimization (SELMA).

Suguang Dou

DTU Wind Energy, Technical University of Denmark,

Frederiksborgvej 399, 4000 Roskilde, Denmark

E-mail: sudou@dtu.dk

ORCID: 0000-0002-8136-9437

Mathias Stolpe

DTU Wind Energy, Technical University of Denmark,

Frederiksborgvej 399, 4000 Roskilde, Denmark

E-mail: matst@dtu.dk

ORCID: 0000-0002-5858-1595
(1999); Jansen et al. (2014); Zhou and Fleury (2016); Peng and Sui (2018); Lüdeker and Kriegesmann (2019); Stolpe (2019); Pollini (2020); Ambrozkiewicz and Kriegesmann (2020). Previous studies have considered complete loss of one arbitrary member in frame structures (Sun et al., 1976; Lüdeker and Kriegesmann, 2019; Stolpe, 2019), or complete loss of one arbitrary element/patch of material in continuum structures (Jansen et al., 2014; Zhou and Fleury, 2016). A few studies have considered the local degradation of the structural geometry or material properties in fail-safe structural optimization (Sun et al., 1976; Achtziger and Bendsøe, 1999; Stolpe, 2019).

Even fewer studies have examined stress constrained failsafe optimization (Sun et al., 1976; Lüdeker and Kriegesmann, 2019). This study presents an analytical study of stress-constrained fail-safe truss optimization considering local degradation of the cross-section area of one arbitrary member. As shown in the following optimization problems, amplified stress in a degraded member may drive the design to an overly conservative design for severe degradation. This is similar to the singular optimum problem for stress constrained topology optimization (Kirsch, 1990; Cheng and Jiang, 1992; Rozvany, 2001). The problematic design-driving stress constraint vanishes when the related member is completely damaged and thus lost, leading to a design with lower objective value. This study was initiated to explain a phenomenon that was observed while numerically solving failsafe optimization problems with stress constraints.

\section{Examples}

In this analytical study, we consider a variant of the classical three-bar truss problem (Kirsch, 1990). The considered structure is displayed in Figure 1. For further details on the analysis and optimization of the three-bar truss example we 
refer to the supplementary material. Similar structures were studied by Zhou and Fleury (2016) and Stolpe (2019) to illustrate the concept and behavior of a fail-safe design. There are three damage scenarios. In each damage scenario, one bar is damaged while the others remain undamaged. The damage model is defined in terms of the degradation of the cross-section area. Such degradation can be caused by e.g. the effects of corrosion in engineering structures. In the $j^{\text {th }}$ damage scenario, the cross section area of bar $j$ is degraded, leading to $a_{j}^{(\gamma)}=a_{j}(1-\gamma)$, where $a_{j}$ denotes the nominal cross-section area of bar $j$, and $\gamma$ denotes the level of degradation. Particularly, when $\gamma=1$, we have $a_{j}^{(\gamma)}=0$, which means that bar $j$ vanishes.

For the three-bar problem presented in Figure 1 the static horizontal load $(F)$, the modulus of elasticity $\left(E_{i}\right)$, the bar lengths $\left(l_{i}\right)$, and the stress limits $\left(\bar{\sigma}_{i}\right.$ and $\left.\underline{\sigma}_{i}\right)$ are

$$
\begin{aligned}
& F=1, l_{1}=l_{3}=\sqrt{2}, l_{2}=1, E_{1}=E_{2}=E_{3}=1, \\
& \bar{\sigma}_{1}=\bar{\sigma}_{2}=\bar{\sigma}_{3}=-\underline{\sigma}_{1}=-\underline{\sigma}_{2}=-\underline{\sigma}_{3}=\bar{\sigma}=1 .
\end{aligned}
$$
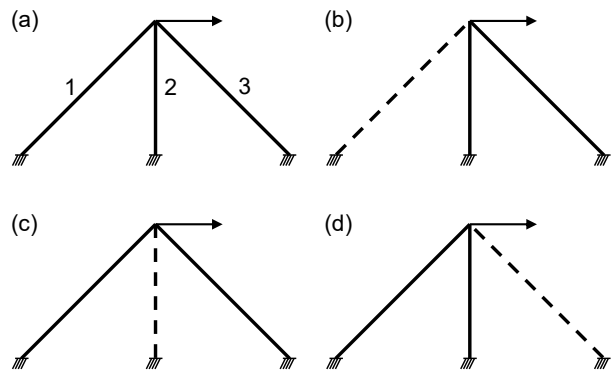

Fig. 1 (a) Ground structure, boundary conditions, and external load for the three-bar truss example used to illustrate the stress singularity issue and the fail-safe optimization problems. (b), (c) and (d) Three damage scenarios with one bar partially or fully damaged. The damage scenarios in (a), (b), (c) and (d) are denoted by $j=0,1,2,3$, respectively.

\subsection{Stress-constrained truss sizing problems}

Design variables The cross-section areas of the three bars are chosen as design variables in the optimization. Variable grouping is applied to reduce the number of design variables to two. Bars 1 and 3 are required to have the same crosssection area, i.e. $a_{1}=a_{3}$. Due to the symmetry, this choice of modelling does not interfere with the objectives to study the feasible sets and the optimal objective values but allows for easier graphical representation of the feasible sets.

Nominal problem and fail-safe problems The nominal problem, i.e. a classical minimum mass truss sizing problem with stress constraints, but without considering any damage scenarios, is

$$
\begin{aligned}
\left.m^{*}(\gamma)\right|_{\gamma=0}= & \min _{\mathbf{a} \in \mathcal{F}} m(\mathbf{a})=2 \sqrt{2} a_{1}+a_{2} \\
& \text { s.t. } \underline{\sigma}_{i} \leq \sigma_{i}(\mathbf{a}) \leq \bar{\sigma}_{i} \quad i \in \mathcal{N}
\end{aligned}
$$

where $\sigma(\mathbf{a})_{i}$ is the axial stress in the $i$ th bar and the set $\mathcal{N}=\{1,2,3\}$. The set $\mathcal{F}=\left\{\mathbf{a} \in \mathbb{R}^{2} \mid a_{i} \geq \underline{a}_{i} \forall i\right\}$, with $\underline{a}_{i}=1 / 2$.

Depending on the level of degradation, two fail-safe problems corresponding to the nominal problem $(P)$ emerge. When the level of degradation $\gamma<1$, all bars remain in the structure and all stress constraints should also be included. This was used in e.g. Sun et al. (1976). The first fail-safe problem corresponding to nominal problem $(P)$ which considers the degradation of a member is

$$
\begin{aligned}
\left.m^{*}(\gamma)\right|_{\gamma \in(0,1)}= & \min _{\mathbf{a} \in \mathcal{F}} m(\mathbf{a}) \\
& \text { s.t. } \underline{\sigma}_{i} \leq \sigma_{i}^{j}(\mathbf{a}) \leq \bar{\sigma}_{i} \forall j, i \in \mathcal{N}
\end{aligned} \quad\left(P_{F S}^{\gamma<1}\right)
$$

where $j=0,1,2,3$ corresponds to the damage scenarios shown in Fig. 1(a), (b), (c), (d), respectively. The stress $\sigma_{i}^{j}$ denotes the stress of bar $i$ in the damage scenario $j$.

When the level of degradation $\gamma=1$, the corresponding stress constraints in the vanished member should be excluded. The second fail-safe problem corresponding to the nominal problem $(P)$ which models the complete loss of a member becomes

$$
\begin{aligned}
\left.m^{*}(\gamma)\right|_{\gamma=1}= & \min _{\mathbf{a} \in \mathcal{F}} m(\mathbf{a}) \\
& \text { s.t. } \underline{\sigma}_{i} \leq \sigma_{i}^{j}(\mathbf{a}) \leq \bar{\sigma}_{i} \forall j, i \in \mathcal{I}_{j} \quad\left(P_{F S}^{\gamma=1}\right)
\end{aligned}
$$

where $\mathcal{I}_{j}=\mathcal{N} \backslash\{j\}$ and it is assumed that the set $\mathcal{I}_{0}=\mathcal{N}$. Though problem $\left(P_{F S}^{\gamma=1}\right)$ and $\left(P_{F S}^{\gamma<1}\right)$ are similar, they differ in the number of stress constraints and the assembly of the stiffness matrices in the damage scenarios.

The feasible sets of problems $\left(P_{F S}^{\gamma<1}\right)$ and $\left(P_{F S}^{\gamma=1}\right)$ thus both contain the feasible set of problem $(P)$. An immediate consequence of this observation is: If all problems have optimal solutions then the optimal objective function values of the fail-safe problems $\left(P_{F S}^{\gamma<1}\right)$ and $\left(P_{F S}^{\gamma=1}\right)$ are greater than the optimal objective function value of the nominal problem $(P)$, i.e. $\left.m^{*}\right|_{\gamma=0} \leq\left. m^{*}\right|_{\gamma \in(0,1)}$ and $\left.m^{*}\right|_{\gamma=0} \leq\left. m^{*}\right|_{\gamma=1}$. Another consequence is the possibility that the feasible set of the nominal problem $(P)$ is non-empty, whereas the feasible sets of (one or both of) the fail-safe problems are empty.

The relationship between the feasible sets of the two failsafe problems $\left(P_{F S}^{\gamma<1}\right)$ and $\left(P_{F S}^{\gamma=1}\right)$ is however less obvious. By examining this small-scale example we discover that the optimal objective function value can jump from $\gamma$ just slightly below one to $\gamma$ equal to one. This is a similar observation to what is seen in stress-constrained truss topology optimization when you include stress constraints also for members that have vanished, see e.g. Sved and Ginos (1968).

Solution of the nominal problem For the nominal case and due to the chosen ground structure, variable linking, and load the stress in the second bar is zero for all feasible design variables, i.e. $\sigma_{2}(\mathbf{a})=0$. The stress in the first bar is the negative of the stress in the third bar, i.e. $\sigma_{1}(\mathbf{a})=-\sigma_{3}(\mathbf{a})$, 
Additionally, the first bar is in tension. It is thus possible to simplify the constraints for the nominal design problem. The only required constraint is $\sigma_{1}(\mathbf{a})=1 /\left(\sqrt{2} a_{1}\right) \leq \bar{\sigma}$. This in turn results in a lower bound on the area $a_{1} \geq 1 /(\sqrt{2} \bar{\sigma})=$ $1 / \sqrt{2}$. Since mass is minimized, the second design variable $a_{2}$ is at the lower bound $\underline{a}_{2}$ in the optimal design. For the nominal problem the optimal design is given by $\left.\mathbf{a}\right|_{\gamma=0}=$ $(1 / \sqrt{2}, 1 / 2)$, and the optimal objective is $\left.m^{*}\right|_{\gamma=0}=2 \frac{1}{2}$.

Solution of the fail-safe problem For the second damage scenario shown in Fig. 1(c), the middle bar is not stressed and the two slanted bars have the same stresses as in the nominal case. There is thus no need to include these constraints in the fail-safe design problem since they are redundant. In order to further simplify the study of the fail-safe problems $\left(P_{F S}^{\gamma<1}\right)$ and $\left(P_{F S}^{\gamma=1}\right)$, we from now on consider problems only involving the first damage scenario shown in Fig. 1(b). Figure 2 illustrates the feasible sets for the fail-safe problems $\left(P_{F S}^{\gamma<1}\right)$ and $\left(P_{F S}^{\gamma=1}\right)$. The figures suggest that for $\gamma$ close to one, the only active constraint is $\sigma_{1}^{1}(\mathbf{a})=\bar{\sigma}$. When $\gamma=1$, the stress constraint $\sigma_{1}^{1}(\mathbf{a})=\bar{\sigma}$ vanishes, leading to a design with lower objective function value than that for $\gamma=0.9$.
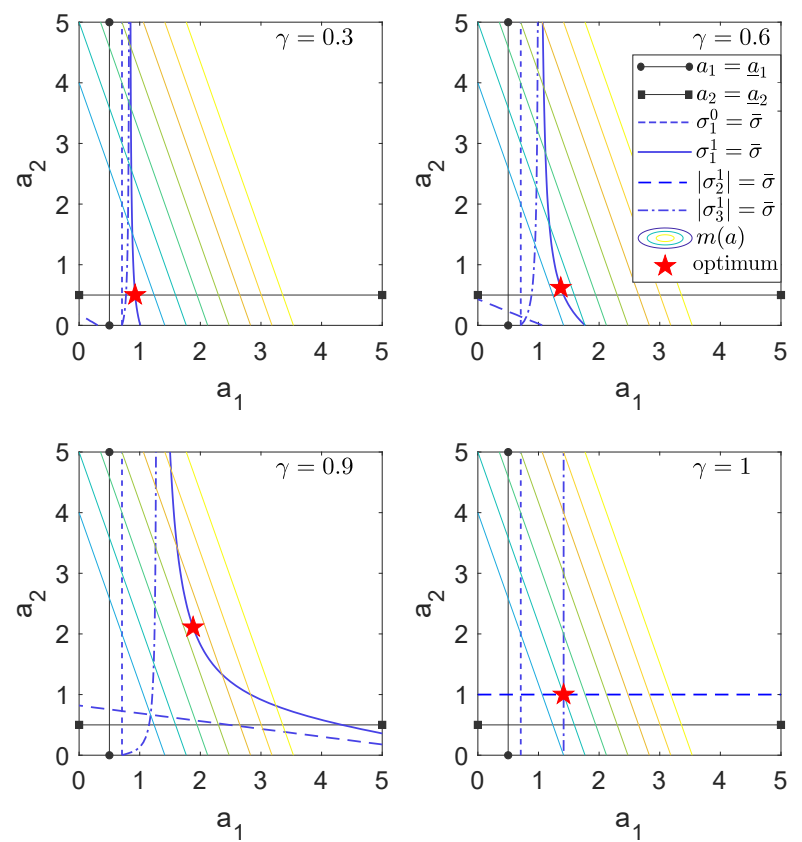

Fig. 2 The feasible sets for the fail-safe problem $\left(P_{F S}^{\gamma<1}\right)$ when $\gamma \in$ $(0,1)$ and the fail-safe problem $\left(P_{F S}^{\gamma=1}\right)$ when $\gamma=1$.

For $\gamma=1$ the optimal solution to the problem $\left(P_{F S}^{\gamma=1}\right)$ is $\left.\mathbf{a}\right|_{\gamma=1}=(\sqrt{2}, 1)$ with optimal objective function value $\left.m^{*}\right|_{\gamma=1}=2 \sqrt{2} \sqrt{2}+1=5$. For $\gamma$ approaching one from below, the optimal design to the problem $\left(P_{F S}^{\gamma<1}\right)$ approaches $(3 / \sqrt{2}, 3)$ with optimal objective value $m^{*}(\gamma)$ approaching 9. The optimal solutions are displayed in Fig. 3. These results illustrate that the problem $\left(P_{F S}^{\gamma<1}\right)$ may result in a more conservative design than problem $\left(P_{F S}^{\gamma=1}\right)$. These more conservative designs occur when the level of degradation is larger than a critical value $\gamma^{\text {ctr }}$. For this example $\gamma^{\text {ctr }} \approx 0.67$. A physical interpretation is that a design with lower objective function value can be found when the level of degradation is allowed to evolve to complete damage.
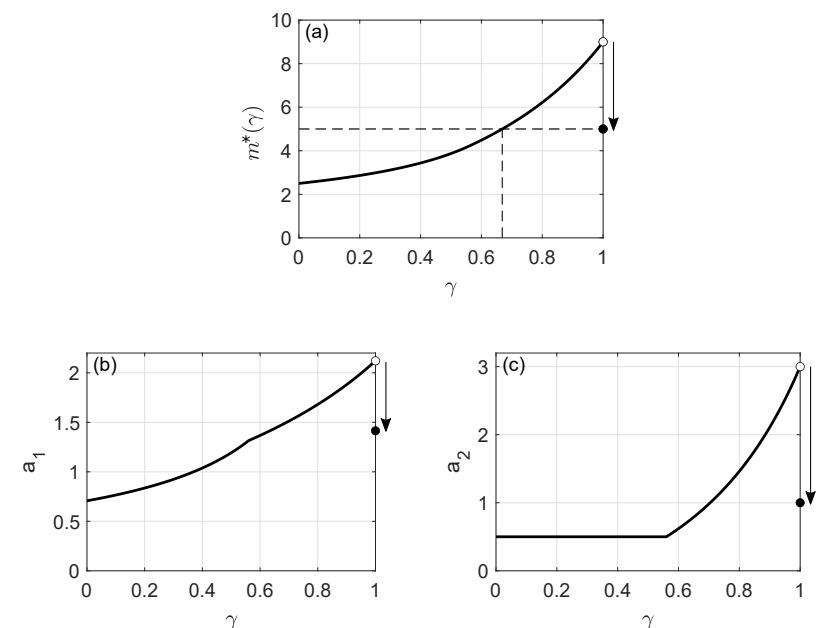

Fig. 3 (a) The optimal objective function values $m^{*}(\gamma)$ for the nominal problem $(P)$ when $\gamma=0$, and fail-safe optimization problem $\left(P_{F S}^{\gamma<1}\right)$ when $\gamma \in(0,1)$ and $\left(P_{F S}^{\gamma=1}\right)$ when $\gamma=1$. (b) and (c): The optimal values for the two design variables $a_{1}$ and $a_{2}$. The solid dots in (a), (b) and (c) denote respectively the optimal objective function value and two design variable values to fail-safe optimization problem $\left(P_{F S}^{\gamma=1}\right)$.

\subsection{Stress analysis of a given structure}

The stress issue encountered in the fail-safe optimization problems, is further interpreted by performing a parametric study of the maximum stress in the structure for a given design against the level of degradation. The cross section areas of the three bars are given as $a_{1}^{(\gamma)}=1-\gamma, a_{2}=$ $a_{3}=1$. Figure 4 shows the maximum stress magnitude in the structure as a function of the level of degradation $\gamma$ in bar 1 . When $\gamma=0$, the maximum stress magnitude is $\sqrt{2} / 2$ occurring in both bar 1 and 3 (point $A$ ). When $\gamma \in(0,1)$, the maximum stress magnitude occurs in the degraded bar 1 . As $\gamma$ approaches 1 , the stress magnitude in bar 1 approaches a finite value of $\sqrt{2}+1$ (point $\mathrm{C}$ ). The curve for $|\sigma|$ is described by $|\sigma|=1 /(\sqrt{2}-\gamma)$. For $\gamma=1$, no stress should be present in bar 1 , since the bar is completely removed. With bar 1 removed, the maximum stress magnitude in the structure is $\sqrt{2}$ in bar 3 (point D). An interpretation of these results imply that a severe degradation can cause a larger stress magnitude than the maximum stress in the structure with the damaged member removed. This amplified maximum stress in the structure vanishes when the level of degradation evolves to complete damage. 


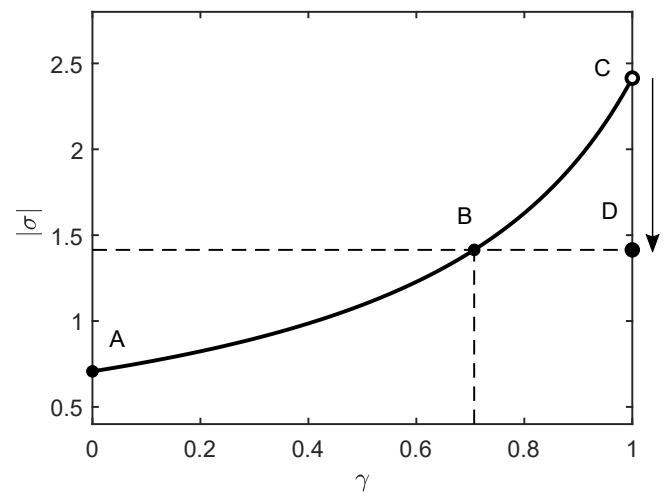

Fig. 4 The maximum stress magnitude in the structure for different levels of degradation $\gamma$. The cross-section areas are $a_{1}^{(\gamma)}=1-\gamma$ and $a_{2}=a_{3}=1$. The key points $(\gamma,|\sigma|)$ are: A $(0, \sqrt{2} / 2), \mathrm{B}(\sqrt{2} / 2, \sqrt{2})$, $\mathrm{C}(1, \sqrt{2}+1), \mathrm{D}(1, \sqrt{2})$

i

\section{Discussion}

We are generally unable to guarantee finding global optimums for the considered problems in practical computations. It is therefore relevant to attempt to study an example with analytical solutions for different levels of degradation. This example thus allows us to distinguish between a property caused by the formulation of the optimization problem or the numerical procedure used to solve it.

The example is based on a small-scale truss sizing problem. The observed stress phenomenon may however also be relevant for other kinds of structural models and for largescale problem instances. The same phenomenon was observed by the authors in numerical studies of stress-constrained fail-safe optimization of tubular frame structures considering thickness degradation.

The example considers extreme situations, (arguably) even for fail-safe design. Allowing a bar to almost disappear as a damage scenario might be unreasonable and unrealistic. It is perhaps more likely that the relative damage parameter is closer to zero than to one and instead more members are considered in the damage scenarios. Hence, in practice the phenomenon outlined by the example might not be observed. However, the observation is worth keeping in mind when modelling and solving fail-safe structural optimization problems with stress constraints.

\section{Conclusion and recommendation}

Analytical solutions to a small truss sizing optimization example demonstrate that the degradation of cross-section area of a truss member may cause more conservative optimal designs for fail-safe optimization problems than complete damage of a truss member.
One could therefore compare the fail-safe designs for degradation of member and complete damage of member, and choose the design that suits the intended applications. This recommendation applies not only to topology optimization problems but also to sizing optimization problems.

\section{Replication of results}

The model descriptions and parameters and the key analytical results are described in the note. Detailed models and analytical results are provided in the supplementary material.

\section{Conflicts of interest}

The authors declare that they have no conflicts of interest.

Acknowledgements We thank our colleague Nicolo Pollini for fruitful discussions on fail-safe design in general and his insightful suggestions on how to improve this manuscript.

\section{References}

Achtziger W, Bendsøe MP (1999) Optimal topology design of discrete structures resisting degradation effects. Struct Optim 17(1):74-78

Ambrozkiewicz O, Kriegesmann B (2020) Density-based shape optimization for fail-safe design. J Comput Des Eng

Cheng G, Jiang Z (1992) Study on topology optimization with stress constraints. Eng Optim 20(2):129-148

Jansen M, Lombaert G, Schevenels M, Sigmund O (2014) Topology optimization of fail-safe structures using a simplified local damage model. Struct Multidiscip Optim 49(4):657-666

Kirsch U (1990) On singular topologies in optimum structural design. Structural Optimization 2(3):133-142

Lüdeker JK, Kriegesmann B (2019) Fail-safe optimization of beam structures. J Comput Des Eng 6(3):260-268

Peng XP, Sui Y (2018) ICM method for fail-safe topology optimization of continuum structures. Chin J Theor Appl Mech 50(3):611-621

Pollini N (2020) Fail-safe optimization of viscous dampers for seismic retrofitting. Earthquake Eng Struct Dyn (Accepted for publication)

Rozvany G (2001) On design-dependent constraints and topologies. Struct Multidiscip Optim 21:164-172

Stolpe M (2019) Fail-safe truss topology optimization. Struct Multidiscip Optim 60(4):1605-1618

Sun P, Arora J, Haug E (1976) Fail-safe optimal design of structures. Eng Optim 2(1):43-53

Sved G, Ginos Z (1968) Structural optimization under multiple loading. Int J Mech Sci 10(10):803-805 
Zhou M, Fleury R (2016) Fail-safe topology optimization.

Struct Multidiscip Optim 54(5):1225-1243 